Supporting Information for

\title{
Comparative Study on Sulfide and Oxide Electrolyte Interfaces with Cathodes in All-Solid-State Battery via
}

\section{First-Principles Calculations}

\author{
Yukihiro Okuno ${ }^{\mathrm{a},{ }^{*}}$, Jun Haruyama ${ }^{\mathrm{b}}$, and Yoshitaka Tateyama ${ }^{\mathrm{c}, \mathrm{d}, *}$ \\ ${ }^{a}$ Research and Development Management Headquarters, FUJIFILM Corporation, 210 Nakanuma, \\ Minamiashigara, Kanagawa 250-0193, Japan, \\ ${ }^{b}$ Institute for Solid State Physics, The University of Tokyo, 5-1-5 Kashiwanoha, Kashiwa, Chiba, \\ 277-8581, Japan,
}

${ }^{c}$ Center for Green Research on Energy and Environmental Materials (GREEN) and International Center for Materials Nanoarchitectonics (MANA), National Institute for Materials Science (NIMS), 1-1 Namiki,

Tsukuba, Ibaraki 305-0044, Japan,

${ }^{d}$ Elements Strategy Initiative for Catalysts \& Batteries, Kyoto University, 1-30 Goryo-Ohara,

Nishikyo-ku, Kyoto 615-8245, Japan. 


\section{S1. Bulk crystals}

The bulk crystal calculations were conducted for $\mathrm{LiCoO}_{2}$, $\mathrm{LCO}$ ), $\beta-\mathrm{Li}_{3} \mathrm{PS}_{4}(\mathrm{LPS}), \quad \gamma-\mathrm{Li}_{3} \mathrm{PO}_{4}(\mathrm{LPO})$, $\mathrm{Li}_{7} \mathrm{La}_{3} \mathrm{Zr}_{2} \mathrm{O}_{12}$ (LLZO), and $\mathrm{Li}_{4} \mathrm{Ti}_{5} \mathrm{O}_{12}$ (LTO) using the DFT+U method. We show the optimized lattice constants and Li vacancy formation energies of the bulk crystals in Table S1.

Table S1. Optimized lattice constant of bulk crystal structures and Li vacancy formation energies (Ev) of $\beta$ - $\mathrm{Li}_{3} \mathrm{PS}_{4}, \mathrm{LiCoO}_{2}, \mathrm{Li}_{4} \mathrm{Ti}_{5} \mathrm{O}_{12}, \gamma-\mathrm{Li}_{3} \mathrm{PO}_{4}$, and $\mathrm{Li}_{7} \mathrm{La}_{3} \mathrm{Zr}_{2} \mathrm{O}_{12}$. The numbers in parentheses are experimental lattice constants.

\begin{tabular}{|c|c|c|c|c|}
\hline Crystal & $\mathrm{a}(\AA)$ & b $\quad(\AA)$ & c $(\AA)$ & $\mathrm{Ev}(\mathrm{eV})$ \\
\hline $\begin{array}{l}\beta-\mathrm{Li}_{3} \mathrm{PS}_{4} \\
\text { (orthorhombic) }^{\mathrm{S} 1}\end{array}$ & $\begin{array}{l}13.02 \\
(13.07)\end{array}$ & $\begin{array}{l}8.161 \\
(8.015)\end{array}$ & $\begin{array}{l}6.234 \\
(6.101)\end{array}$ & $2.9-3.2$ \\
\hline $\begin{array}{l}\mathrm{LiCoO}_{2} \\
\text { (hexagonal) }^{\mathrm{S} 2}\end{array}$ & $\begin{array}{l}2.835 \\
(2.815)\end{array}$ & -- & $\begin{array}{l}14.04 \\
(14.05)\end{array}$ & 4.0 \\
\hline $\begin{array}{l}\mathrm{Li}_{4} \mathrm{Ti}_{5} \mathrm{O}_{12} \\
(\text { cubic })^{\mathrm{S} 3}\end{array}$ & $\begin{array}{l}8.419 \\
(8.357)\end{array}$ & - & - & $5.0-5.1$ \\
\hline $\begin{array}{l}\gamma-\mathrm{Li}_{3} \mathrm{PO}_{4} \\
\text { (orthorhombic) }^{\mathrm{S} 4}\end{array}$ & $\begin{array}{l}10.53 \\
(10.49)\end{array}$ & $\begin{array}{l}6.14 \\
(6.12)\end{array}$ & $\begin{array}{l}4.95 \\
(4.93)\end{array}$ & $5.1-5.2$ \\
\hline $\begin{array}{l}\mathrm{Li}_{7} \mathrm{La}_{3} \mathrm{Zr}_{2} \mathrm{O}_{12} \\
\text { (cubic) }^{\mathrm{S5}}\end{array}$ & $\begin{array}{l}13.03 \\
(12.97)\end{array}$ & - & - & $3.5-3.6$ \\
\hline
\end{tabular}

\section{S2. Interface quantities}

The interface calculations were carried out so as to make the misfit parameter as small as possible. The surface slab $A$ with area $S_{A}$ is compressed or stretched to lock in the surface $B$ with area $\mathrm{S}_{B}$. The $\mathrm{S}_{\mathrm{A}-\mathrm{B}}$ is the overlap between the slabs $\mathrm{A}$ and $\mathrm{B}$. Then, the misfit parameter $\mu$ is defined as ${ }^{\mathrm{S} 6-\mathrm{S} 7}$

$$
\mu=1-2 \mathrm{~S}_{\mathrm{A}-\mathrm{B}} /\left(\mathrm{S}_{\mathrm{A}}+\mathrm{S}_{\mathrm{B}}\right) .
$$

We define the adhesion energy $\mathrm{W}_{\mathrm{ad}}$ as

$$
\mathrm{W}_{\mathrm{ad}}=\left(\mathrm{E}^{\text {tot }}{ }_{\mathrm{A}}+\mathrm{E}^{\mathrm{tot}} \mathrm{B}^{-} \mathrm{E}^{\mathrm{tot}}{ }_{\mathrm{A} / \mathrm{B}}\right) / \mathrm{S}
$$

where $\mathrm{E}^{\text {tot }} \mathrm{A}$ and $\mathrm{E}^{\text {tot }} \mathrm{B}$ are the total energies of relaxed isolated slabs, respectively, $\mathrm{E}^{\text {tot }} \mathrm{A} / \mathrm{B}$ is the total energy of the $\mathrm{A} / \mathrm{B}$ interface system, and $\mathrm{S}$ is the interface area ${ }^{\mathrm{S}}$. 


\section{S3. LCO/LPS interface}

One of slab models of LCO(104)/LPS(010) and LCO(110)/LPS(010) interface we calculated are shown in Figure.S.1. The LCO(104)/LPS(010) slab model is composed of 696 atoms including 506 and 192 atoms in LCO and LPS phase, respectively, and LCO(110)/LPS(010) slab model is composed of 756 atoms including 480 and 256 atoms in LCO and LPS phase, respectively. We had calculated 8 and 4 slab models for LCO(104)/LPS(010) and LCO(110)/LPS(010) interface, respectively, by moving one side of the slab model to lateral direction for each solid-solid interface structure. The typical atomic structure at the LCO-LPS boundary is common to all the calculated slab models. Considering the high elastic modulus of LCO, the lattice constants of LPS is set to that of the LCO for LCO/LPS interfaces. The misfit parameters of $\operatorname{LCO}(104) / \operatorname{LPS}(010)$ and LCO(110)/LPS(010) are 8.9 and $4.6 \%$, respectively. In Table S2, we show the misfit parameters and the repeated number of LCO and LPS units which construct the interface models. The LCO(104), LCO(110), LPS(010) units are $2.815 \times 5.700 \times 8.012$ (18 atom), $2.815 \times 14.04 \times 2.835$ (24 atom), and $13.02 \times 8.161 \times 6.234$ (32 atom) $\AA^{3}$ orthorhombic cell, respectively.

(a)
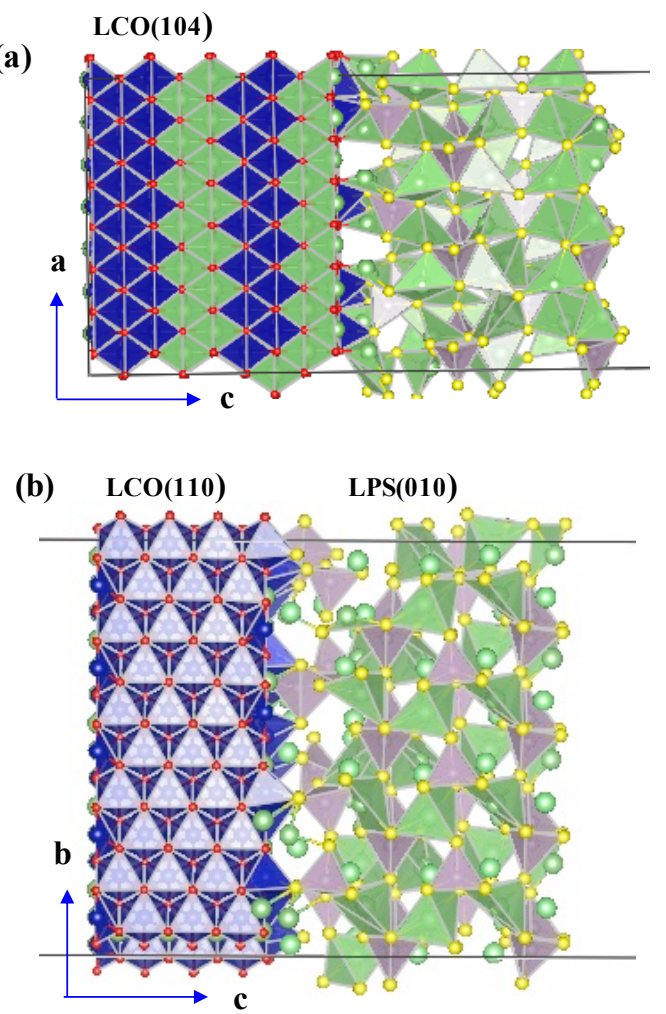
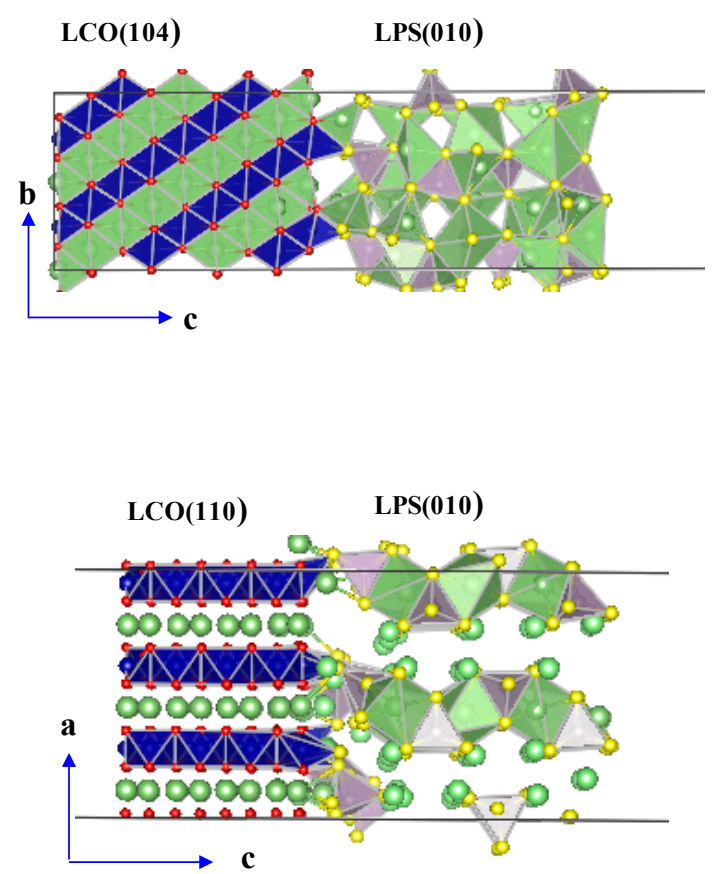

Figure S1. Top and side views of slab models of (a) $\operatorname{LCO}(104) / \operatorname{LPS}(010)$ and (b) LCO(110)/LPS(010). 
Table S2. The repeated numbers of LCO and LPS units in the slab model and the misfit parameter ( $\mu$ ) of the calculated interface.

\begin{tabular}{|l|l|l|l|}
\hline Interface model & $\begin{array}{l}\mathrm{LCO}(104) \text { or } \\
\mathrm{LCO}(111) \text { unit }\end{array}$ & LPS $(010)$ unit & $\mu(\%)$ \\
\hline $\mathrm{LCO}(104) / \mathrm{LPS}(010)$ & $7 \times 2 \times 2$ & $1 \times 2 \times 3$ & 8.9 \\
\hline $\mathrm{LCO}(110) / \mathrm{LPS}(010)$ & $5 \times 1 \times 4$ & $1 \times 2 \times 4$ & 4.6 \\
\hline
\end{tabular}

We show the PDOS of surface Co atoms of LCO(104)/LPS(010) interface in Fig S2. The conduction band bottom is composed by the in-gap state from surface $\mathrm{Co}$ atoms which formed $\mathrm{CoO}_{5}$ quadrangular cone on the LCO surface at the LCO(104)/LPS(010) boundary. Even with the same outermost Co atom on LCO surface, PDOS of Co atoms that formed by attracting S atom in LPS phase at the LCO(104)/LPS(010) boundary do not show the in-gap state.

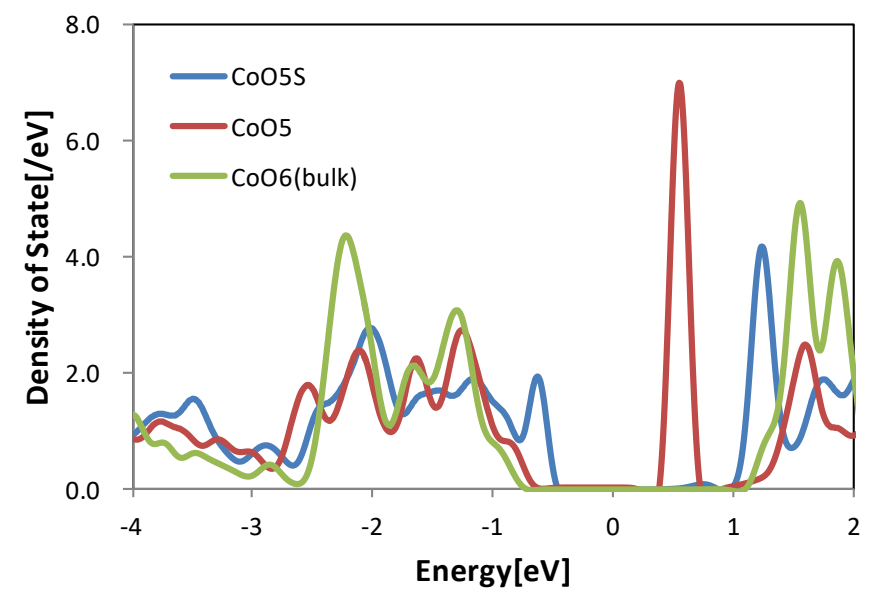

Figure S2. PDOS of Co atoms in LCO(104)/LPS(010) interface. Blue line: Co atom of $\mathrm{CoO}_{5} \mathrm{~S}$ octahedron at the $\mathrm{LCO}(104) / \mathrm{LPS}(010)$ boundary ; red line: $\mathrm{Co}$ atom of $\mathrm{CoO}_{5}$ quadrangular cone on the LCO surface at the LCO(104)/LPS(010) boundary; green line: Co atoms of $\mathrm{CoO}_{6}$ octahedron in the center of LCO slab. We set zero reference energy as the center of the band gap.

Fig. S3 show the Li sites that we have calculated Li vacancy formation energies in the most stable and the highest energy models of LCO(104)/LPS(010) and LCO(110)/LPS(010) interfaces. Table S3 are $\mathrm{Li}$ vacancy formation energies $\left(E_{f}\left(V_{\mathrm{Li}_{j}}\right)\right)$ corresponding to the Li sites of Fig.S3 
(a)

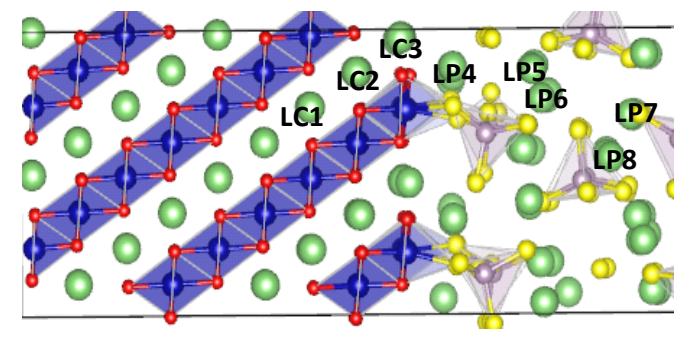

(b)

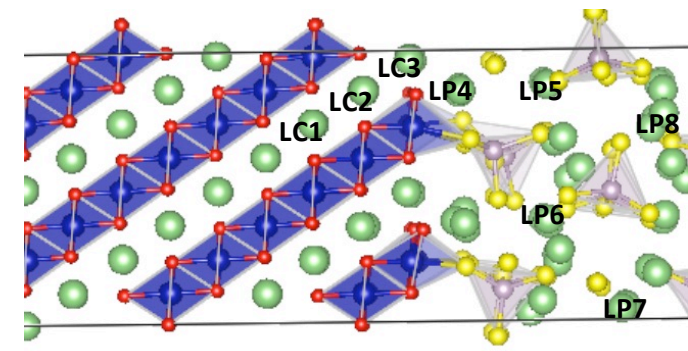

(c)

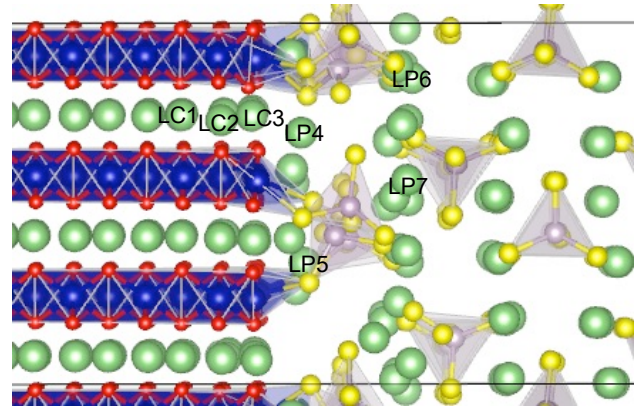

(d)

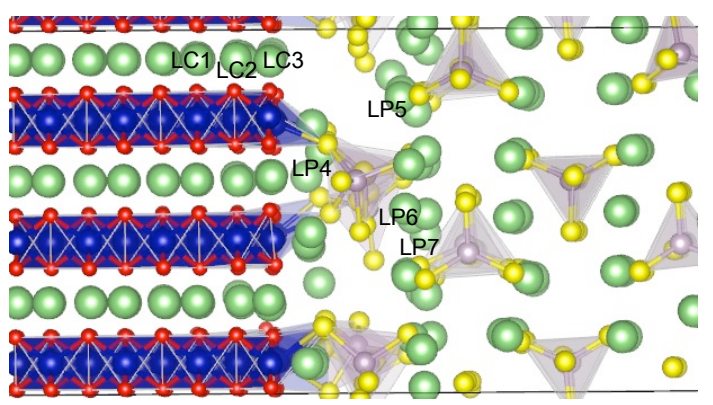

Figure S3. Calculated interface structures with the lowest energies (a) and the highest energy (b) models of LCO(104)/LPS(010) interface, and the sites where we calculated Li vacancy formation energy $\left(E_{f}\left(V_{\mathrm{Li}_{j} j}\right)\right)$. Structure of (c) and (d) are the lowest and the highest energy structures of LCO(110)/LPS(010) interfaces, respectively, and the sites where we calculated Li vacancy formation energy $\left(E_{f}\left(V_{\mathrm{Li}_{j} j}\right)\right)$. 
Table S3. Li vacancy formation energies (eV) of each Li site. The symbols of Li sites (LC1 LP8 ) are corresponding to Figure S3. (a) and (b) are the most stable and the highest energy models of LCO(104)/LPS(010), respectively, and (c) and (d) are those of LCO(110)/LPS(010), in our sampling structures.

\begin{tabular}{|l|l|l|l|l|l|l|l|l|}
\hline & LC1 & LC2 & LC3 & LP4 & LP5 & LP6 & LP7 & LP8 \\
\hline (a) LCO(104)/LPS(010) & 3.37 & 3.41 & 2.41 & 2.43 & 2.17 & 2.40 & 2.15 & 2.27 \\
\hline (b) LCO(104)/LPS(010) & 3.48 & 3.18 & 2.21 & 2.20 & 2.22 & 1.23 & 1.48 & 1.61 \\
\hline (c) LCO(110)/LPS(010) & 2.98 & 3.30 & 2.75 & 2.51 & 2.84 & 1.97 & 1.86 & - \\
\hline (d) LCO(110)/LPS(010) & 3.23 & 3.73 & 3.01 & 2.95 & 2.76 & 2.30 & 1.25 & - \\
\hline
\end{tabular}

In the LCO(104)/LPS(010) interface model of (a) and (b) of Table S3, LP4 is a Li site that is strongly adsorbed by interfacial oxygen on LCO surface, therefore, it has a relatively high defect formation energy. LC3 is outermost Li atom in LCO surface show rather low Li vacancy formation energy compared to other Li atoms like LC1 and LC2 in LCO phase. LP5 and LP8 of model (a), which have low defect formation energies, are mobile $\mathrm{Li}$ sites in the original $\beta-\mathrm{Li}_{3} \mathrm{PS}_{4}$ crystal. LP6 LP8 site in model (b) have low vacancy formation energies that are less than $2.0 \mathrm{eV}$. In particular, LP6 and LP7 have vacancy formation energy below $1.5 \mathrm{eV}$ and the tendency of generation of Li site with such low vacancy formation energy are seen with rather high energy interface models compared to most stable interface structure. On the other hand in LCO(110)/LPS(010) interface model, Li sites with vacancy formation energy below $2.0 \mathrm{eV}$ are seen even in most stable interface model ((c) of Table S3). The tendency of increasing the number of Li site with vacancy formation energy below $1.5 \mathrm{eV}$ in rather high energy interface model are also seen in $\operatorname{LCO}(110) / \mathrm{LPS}(010)$ interface.

Here, we compared the structural dependence of mutual cation exchange energies. We show the Co $\leftrightarrow \mathrm{P}$ exchange energy of most stable and highest energy interface structure in Fig S4. Fig.S4 show that the rather high energy interface structure show the large exothermic energy for $\mathrm{Co} \leftrightarrow \mathrm{P}$ exchange excluding Co1 $\leftrightarrow \mathrm{P} 1$. The high energy interface would promote the mutual exchange reaction energy if it realized as a meta-stable state. 
(a)

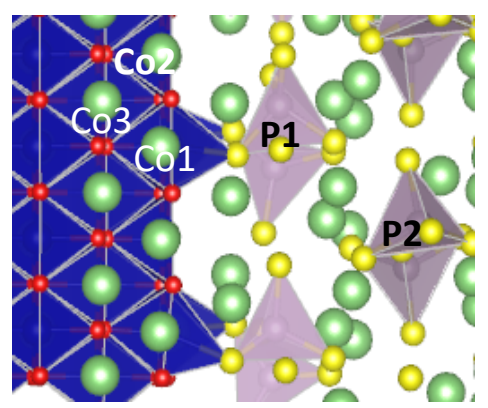

(c)

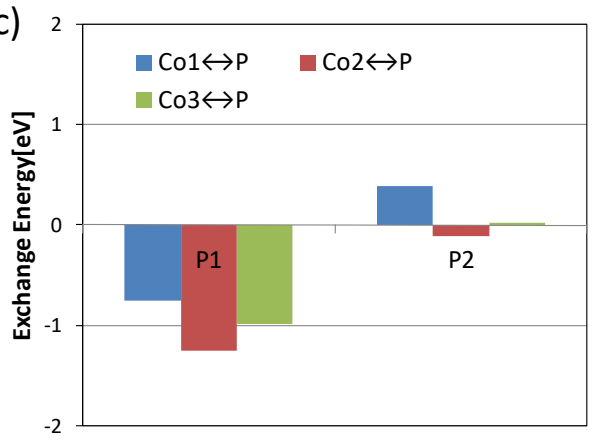

(b)

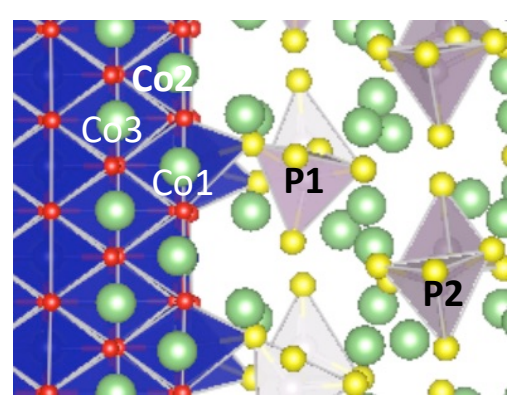

(d)

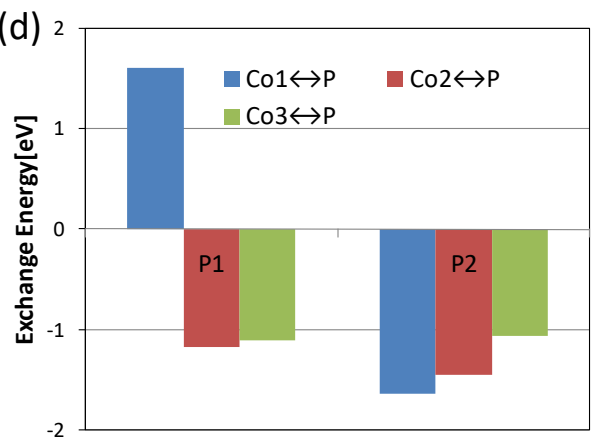

Figure S4. Calculated interface structures with the lowest energy (a) and the highest energy of LCO(104)/LPS(010) interface model. Co $\leftrightarrow \mathrm{P}$ exchange energy for the interface with the lowest energy (c) and that with highest energy (d). The indexes of Co1 Co3 and P1 P3 in (c) and (d) are corresponding to (a) and (b), respectively.

In Fig.S5, we show the LCO(104)/LPS(010) interface with Co and P (Co $\leftrightarrow$ P) mixing, and Table $\mathrm{S} 4$ shows the Li vacancy formation energy before and after Co $\leftrightarrow \mathrm{P}$ mixing reaction. We can see from Table S4 that Li vacancy formation energies are lowered by $\mathrm{Co} \leftrightarrow \mathrm{P}$ mixing reaction.

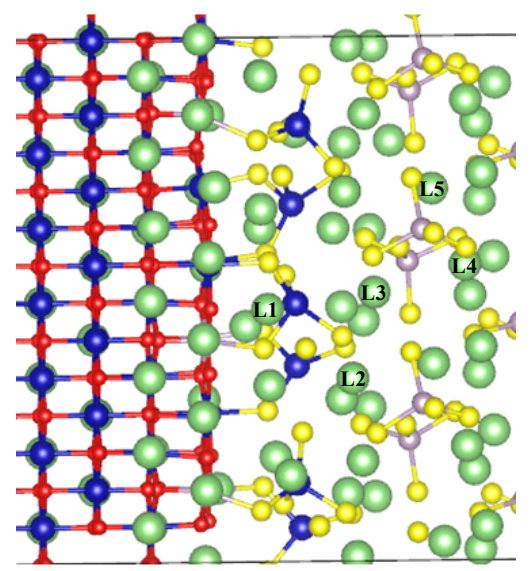

Figure S5. The Li sites where we calculated Li vacancy formation energy after $\mathrm{Co} \leftrightarrow \mathrm{P}$ cation mixing had occurred. Six Co atoms on LCO surface are exchanged to the P atoms in first layer in LPS phase. The model before exchange of cation are the most stable model of LCO(104)/LPS(010). 
Table S4. Li vacancy formation energy $(\mathrm{eV})$ before and after $\mathrm{Co}$ and $\mathrm{P}$ exchange reaction in LCO(104)/LPS(010) interface. L1 L4 are Li site we calculated Li vacancy formation energy and their locations are shown in Fig.S4.

\begin{tabular}{|l|l|l|l|l|l|}
\hline & L1 & L2 & L3 & L4 & L5 \\
\hline Before mixing & 2.40 & 2.18 & 2.40 & 2.27 & 2.14 \\
\hline After mixing & 1.39 & 1.69 & 2.11 & 1.12 & 1.48 \\
\hline
\end{tabular}
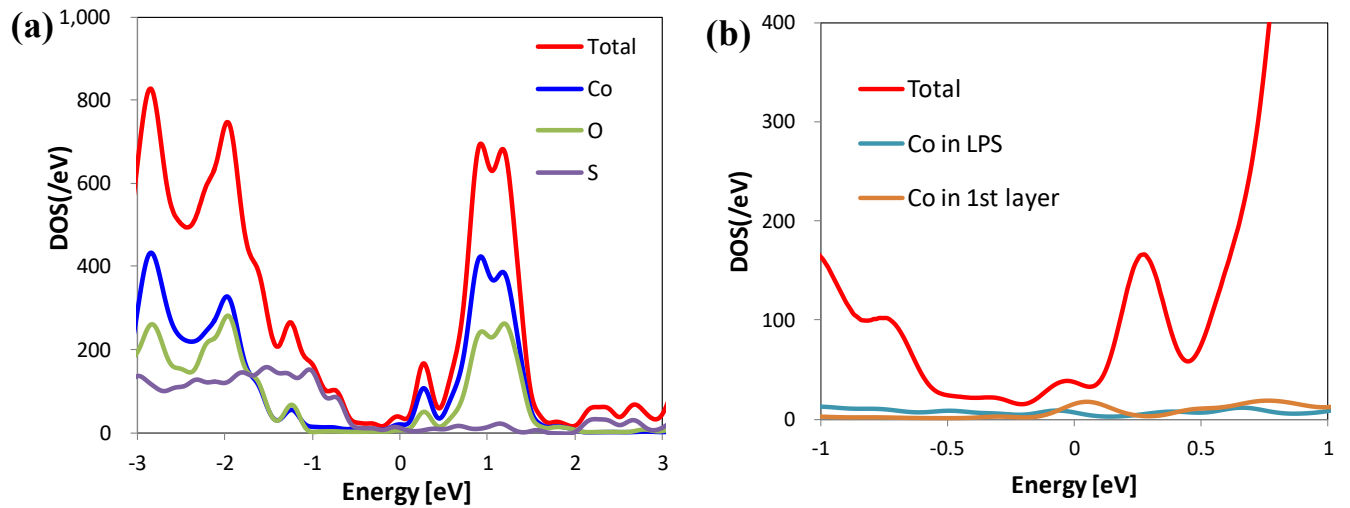

Figure S6. (a): Total DOS (red) and PDOS of Co (blue), O (green), S (purple) atoms in LCO(104)/LPS(010) interface with Co $\leftrightarrow$ P cation mixing corresponding to Fig.S5. (b): Total DOS (red), PDOS of Co atoms in LPS layer (light blue) and PDOS of Co atom in first LCO layer facing to the LPS slabs (orange). The zero reference energy is set to Fermi level of LCO(104)/LPS(010) interface slab.

In Fig.S6, we show the total and partial density of states of $\mathrm{Co}, \mathrm{O}$, and $\mathrm{S}$ atoms in LCO(104)/LPS(010) slab model with Co $\leftrightarrow$ P cation mixing corresponding to Fig.S5. In particular for Co atoms, we show the PDOS of Co atoms located in LPS and the first LCO layer facing LPS slabs. From Fig.S6, we can see the in-gap states around Fermi level are increased by $\mathrm{Co} \leftrightarrow \mathrm{P}$ cation mixing compared to the pristine LCO(104)/LPS(010) interface. The in-gap states around Fermi level are mainly composed of Co atoms at the interface of LCO surface. 


\section{S4. LCO/LPO interface}

One of slab models of $\mathrm{LCO}(104) / \mathrm{LPO}(001)$ and $\mathrm{LCO}(104) / \mathrm{LPO}(010)$ interface we calculated are given in Fig. S7. The $\mathrm{LCO}(104) / \mathrm{LPO}(001)$ slab model is composed of 888 atoms including 504 and 384 atoms in LCO and LPO phase, respectively, and LCO $(104) / \mathrm{LPO}(010)$ slab model is composed of 760 atoms including 504 and 256 atoms in LCO and LPO phase, respectively. We show the one of LCO(110)/LPO(010) interface slab models in Fig.S8. The calculated LCO(110)/LPO(010) slab model is composed of 384 atoms including 192 and 192 atoms in LCO and LPO phase, respectively. The average of two lattice constants is taken for the LCO/LPO interfaces. The misfit parameters of $\mathrm{LCO}(104) / \mathrm{LPO}(001), \mathrm{LCO}(104) / \mathrm{LPO}(010)$, and $\mathrm{LCO}(110) / \mathrm{LPO}(010)$ are $7.0,3.9$ and $6.2 \%$, respectively. In Table S5, we show the misfit parameters and the repeated number of LCO and LPO units which construct the interface models. The LCO(104), LCO(110), LPO(001), LPO(010) units

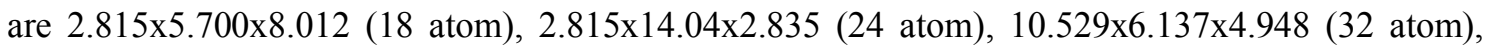
and $10.529 \times 6.137 \times 4.948 \AA^{3}$ orthorhombic cell, respectively.

Table S5. The repeated numbers of LCO and LPO units in the slab model and the misfit parameter ( $\mu$ ) of the calculated interface.

\begin{tabular}{|l|l|l|l|}
\hline Interface model & $\begin{array}{l}\mathrm{LCO}(104) \text { or } \\
\mathrm{LCO}(110) \text { unit }\end{array}$ & $\begin{array}{l}\mathrm{LPO}(001) \text { or } \\
\mathrm{LPO}(010) \text { unit }\end{array}$ & $\mu(\%)$ \\
\hline $\mathrm{LCO}(104) / \mathrm{LPO}(001)$ & $7 \times 2 \times 2$ & $2 \times 2 \times 3$ & 7.0 \\
\hline $\mathrm{LCO}(104) / \mathrm{LPO}(010)$ & $7 \times 2 \times 2$ & $1 \times 2 \times 4$ & 3.9 \\
\hline $\mathrm{LCO}(110) / \mathrm{LPO}(010)$ & $2 \times 1 \times 4$ & $1 \times 2 \times 3$ & 6.2 \\
\hline
\end{tabular}


(a)
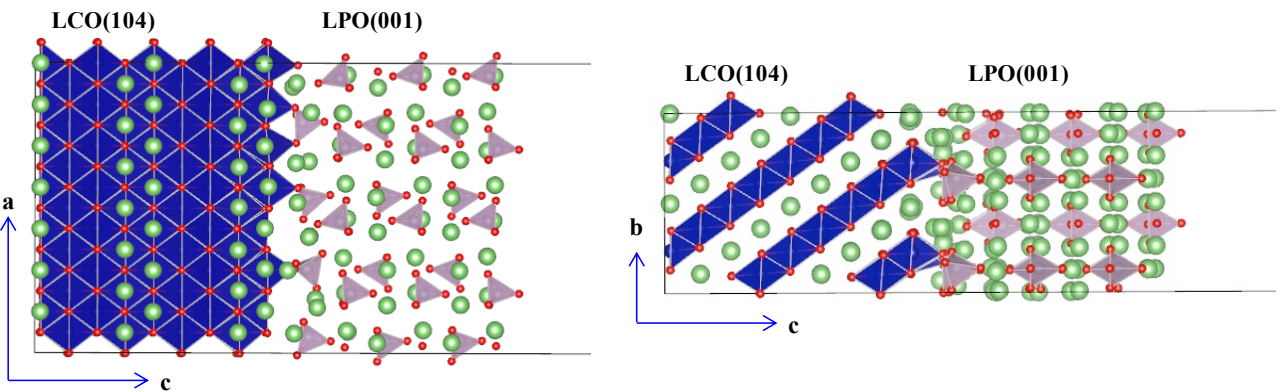

(b)
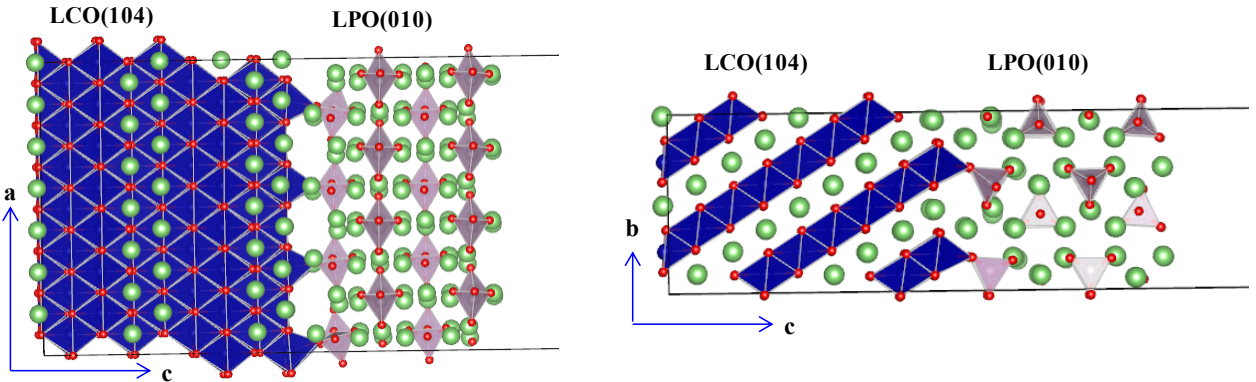

Figure S7. Top and side views of slab models of (a) $\operatorname{LCO}(104) / \operatorname{LPO}(001)$ and (b) $\mathrm{LCO}(104) / \mathrm{LPO}(010)$.
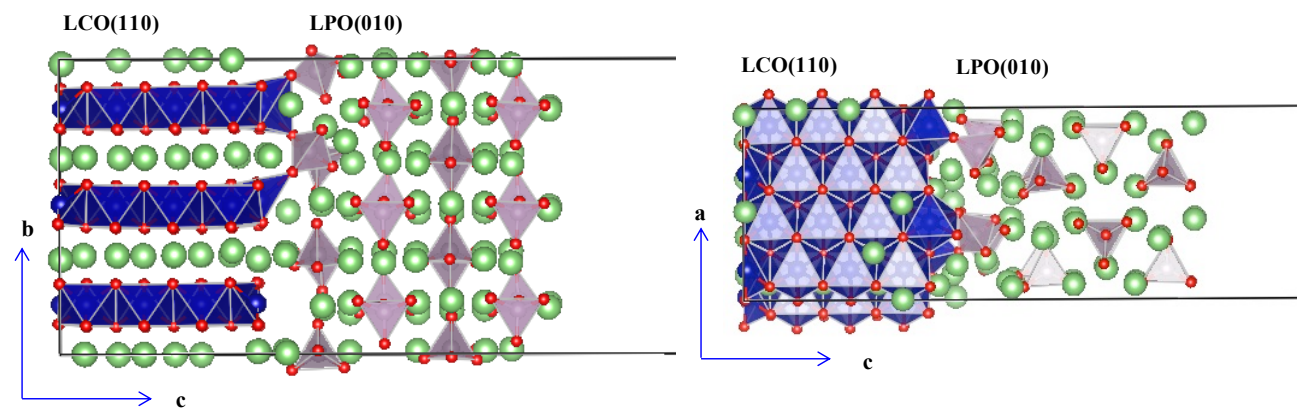

Figure S8. Top and side views of slab models of $\operatorname{LCO}(110) / \mathrm{LPO}(010)$.

Fig.S9 show the Li sites that we have calculated Li vacancy formation energies in one of slab models of $\mathrm{LCO}(104) / \mathrm{LPO}(001)$ interface. Table S6 are Li vacancy formation energies corresponding to the Li sites of Fig.S9. From Table S6, we can see that difference of Li vacancy formation energies in LCO phase is small. Even at the Li site on LCO surface, Li vacancy formation energy did not decrease as much as in the case of $\operatorname{LCO}(104) / \operatorname{LPS}(010)$ interface. The reason of this result would be the outermost $\mathrm{Co}$ atom forms $\mathrm{CoO}_{6}$ octahedron with $\mathrm{O}$ atoms in $\mathrm{LPO}$ side and the environment of $\mathrm{Li}$ site is similar to that of bulk phase of LCO. The Li vacancy formation energies in LPO region are more than $2 \mathrm{eV}$ smaller than that of bulk region of LPO (about $5.0 \mathrm{eV}$ in bulk) in this slab model shown in Table S6. It reflects the band offset between LCO and LPO interface. 
(a)

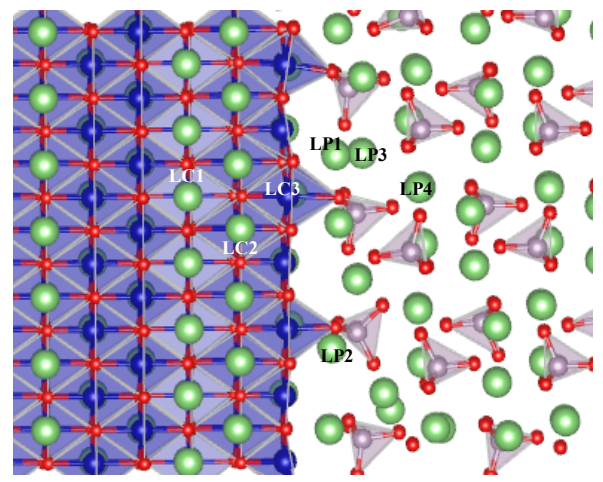

(b)

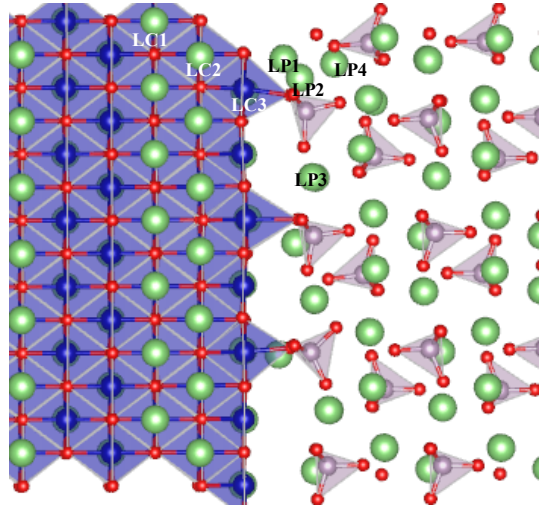

Figure S9. Calculated interface structures with the lowest (a) and the highest energy (b) models of $\mathrm{LCO}(104) / \mathrm{LPO}(001)$ interface, and the sites where we calculated Li vacancy formation energy.

Table S6. Li vacancy formation energies $(\mathrm{eV})$ of each Li site where LC1 LC3 are Li site in LCO and LP1 LP4 are those in LPO. The symbols of Li sites (LC1 LP4) are corresponding to (a) and (b) in Fig. S9 are the lowest and the highest energy models in our sampling calculation, respectively.

\begin{tabular}{|l|l|l|l|l|l|l|l|}
\hline & LC1 & LC2 & LC3 & LP1 & LP2 & LP3 & LP4 \\
\hline (a) & 3.51 & 3.53 & 3.52 & 2.46 & 2.78 & 2.56 & 2.39 \\
\hline (b) & 3.41 & 3.64 & 3.30 & 3.06 & 2.83 & 2.58 & 2.63 \\
\hline
\end{tabular}

As shown in Table S6, the large difference of Li vacancy formation energies are not seen between the most stable and the highest energy slab models we calculated. However, other meta-stable structures we calculated show the generation of Li sites with low Li vacancy formation energy as shown in Fig8 of main text. Fig.S9 (a) and Table S6 indicates that, on the LCO side of the $\mathrm{LCO}(104) / \mathrm{LPO}(001)$ interface, there is no significant difference in $E_{f}\left(\mathrm{~V}_{\left.\mathrm{Li}_{j}\right)}\right)$, even at the Li site on the outermost surface of the LCO. The vacancy-formation energies do not decrease as much as in the case of $\operatorname{LCO}(104) / \operatorname{LPS}(010)$. This result suggests that the outermost Co atoms form $\mathrm{CoO}_{6}$ octahedrons by sharing the $\mathrm{O}$ atoms of the $\mathrm{PO}_{4}$ tetrahedrons in the LPO region and create an environment close to the bulk LCO state.

We show the distribution of $\mathrm{Li}$ sites divided by their vacancy formation energies at the LCO(110)/LPS(001) interface in Fig.S10. The energy difference per interface area between the most stable and the highest energy states among the calculated slab models are $0.019 \mathrm{eV} / \AA^{2}$. Compared 
with the $\operatorname{LCO}(104) / \operatorname{LCO}(010)$ case, the percentage of Li site with low vacancy formation energy is increased and it may be related to the strong chemical activity of $\operatorname{LCO}(110)$ surface compared to that of $\mathrm{LCO}(104)$.

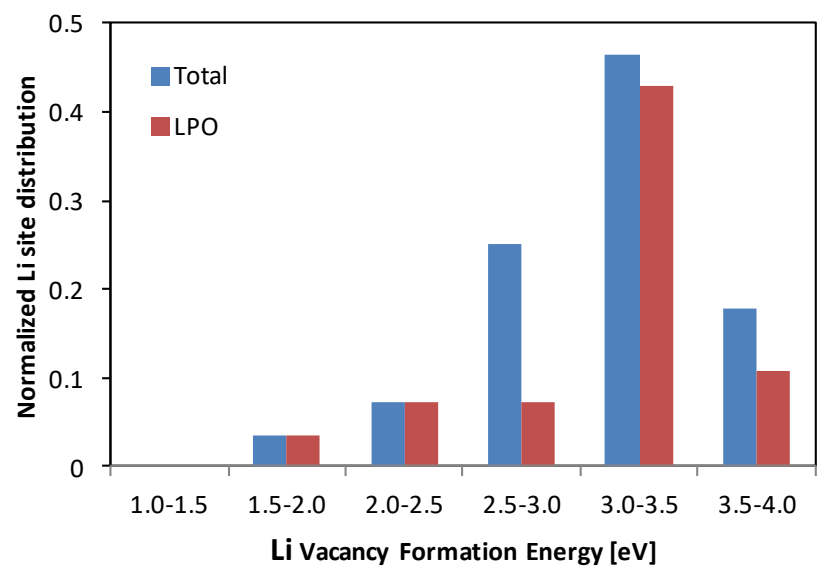

Figure S10. The distributions of $\mathrm{Li}$ sites divided by their $\mathrm{Li}$ vacancy formation energies at $\mathrm{LCO}(110) / \mathrm{LPO}(010)$ interface. The vacancy formation energies of $28 \mathrm{Li}$ sites in 4 different interface structures are calculated. The distributions are normalized by the total number of calculated Li sites. The blue bar graphs denote distribution of all the calculated Li sites in LCO/LPO and red bar graphs are those of calculated Li sites located in LPO region.

Fig.S11 show the Li sites that we have calculated $\mathrm{Li}$ vacancy formation energies in one of slab models of LCO(110)/LPO(010) interface which has the most stable energy in our calculated 4 slab models of $\mathrm{LCO}(110) / \mathrm{LPO}(010)$. Table $\mathrm{S} 7$ are $\mathrm{Li}$ vacancy formation energies corresponding to the $\mathrm{Li}$ sites of Fig.S11.

(a)

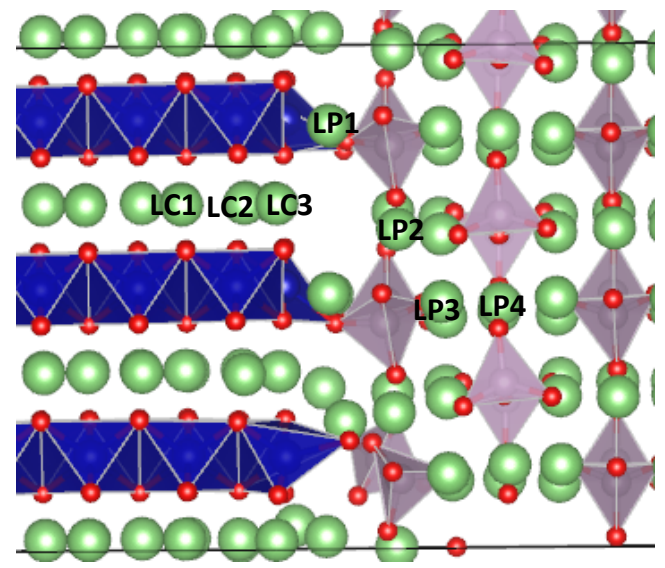

(b)

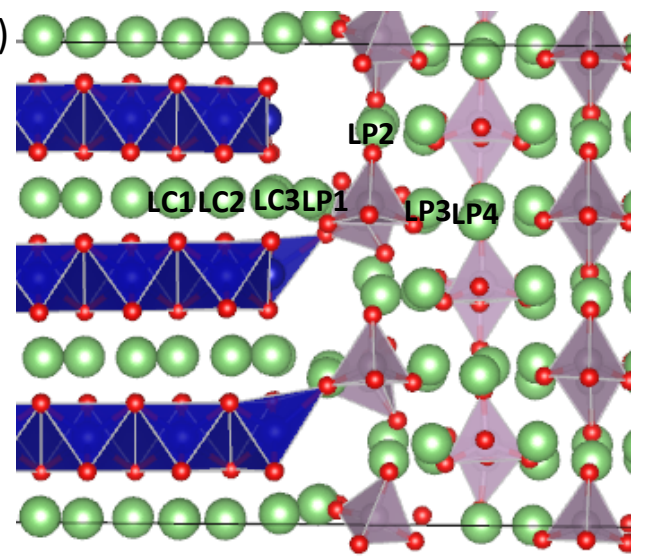

Figure S11. (a) The most stable and (b) the highest energy $\operatorname{LCO}(110) / \mathrm{LPO}(010)$ interface structures we calculated, and the Li sites where we calculated Li vacancy formation energy. 
Table S7. Li vacancy formation energies $(\mathrm{eV})$ of each Li site where LC1 LC3 are Li site in LCO and LP1 LP4 are those in LPO. The symbols of Li sites (LC1 LP4 ) are corresponding to (a) and (b) in Fig. S11 are the most stable and the highest energy models in our sampling calculation, respectively.

\begin{tabular}{|l|l|l|l|l|l|l|l|}
\hline & LC1 & LC2 & LC3 & LP1 & LP2 & LP3 & LP4 \\
\hline (a) & 3.05 & 3.57 & 2.89 & 3.51 & 3.08 & 3.37 & 3.11 \\
\hline (b) & 3.04 & 3.23 & 2.98 & 3.48 & 2.18 & 3.14 & 2.80 \\
\hline
\end{tabular}

Table S7 show the rather high energy structure (b) has Li site with low vacancy formation energy (LP2) compared to the most stable structure (a). This results show the meta-stable state with rather high energy becomes the dynamic Li depletion layer and it may be related that the low interfacial resistance at $\mathrm{LCO} / \mathrm{LPO}$ interface can be observed only in the damage-less interface. 


\section{S5. LCO/LLZO interface}

First, we show the DOS of bulk LLZO crystal in Fig.S11

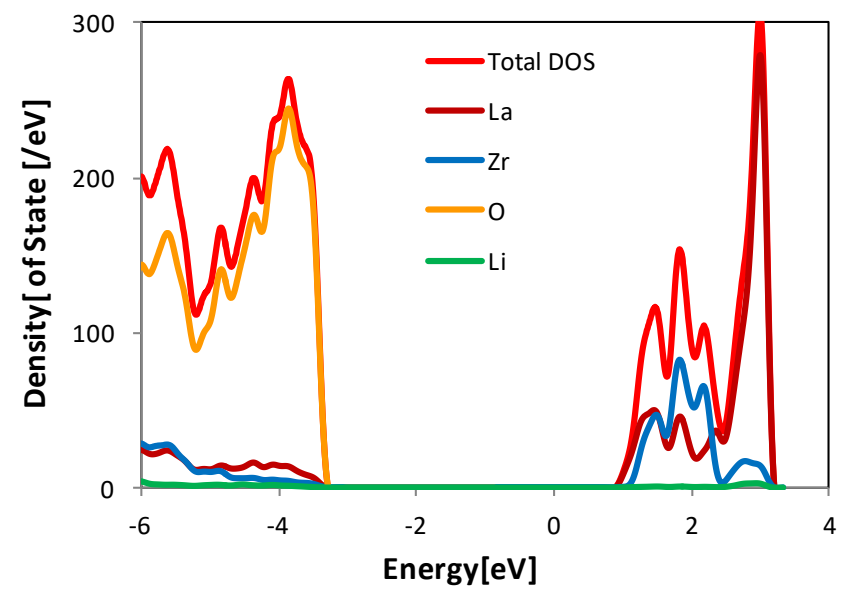

Figure S12. Total and PDOS of bulk LLZO crystal.

From Fig.S12, we can see the valence band of LLZO is mainly composed of $\mathrm{O}$ and the conduction is mainly composed of $\mathrm{Zr}$, La including hybridization with $\mathrm{O}$.

One of slab models of LCO(104)/LLZO(001) interface we calculated are given in Fig. S13. The LCO(104)/LLZO(001) slab model is composed of 448 atoms including 256 and 192 atoms in LCO and LLZO phase, respectively. The misfit parameter of $\operatorname{LCO}(104) / \operatorname{LZZO}(001)$ interface is $13 \%$.

In Table S8, we show the misfit parameters and the repeated number of LCO and LLZO units which construct the interface models. The LCO(104) and LLZO (001) units are 2.815x5.700x8.012 (16 atom) $\AA^{3}$ orthorhombic and cell $13.03 \times 13.03 \times 13.03 \AA^{3}$ (192 atom) cubic cell, respectively.

Table S8. The repeated numbers of LCO and LLZO units in the slab model and the misfit parameter $(\mu)$ of the calculated interface.

\begin{tabular}{|l|l|l|l|}
\hline Interface model & $\mathrm{LCO}(104)$ unit & LLZO $(001)$ unit & $\mu(\%)$ \\
\hline $\mathrm{LCO}(104) / \mathrm{LLZO}(001)$ & $4 \times 2 \times 2$ & $1 \times 1 \times 1$ & 13.0 \\
\hline
\end{tabular}
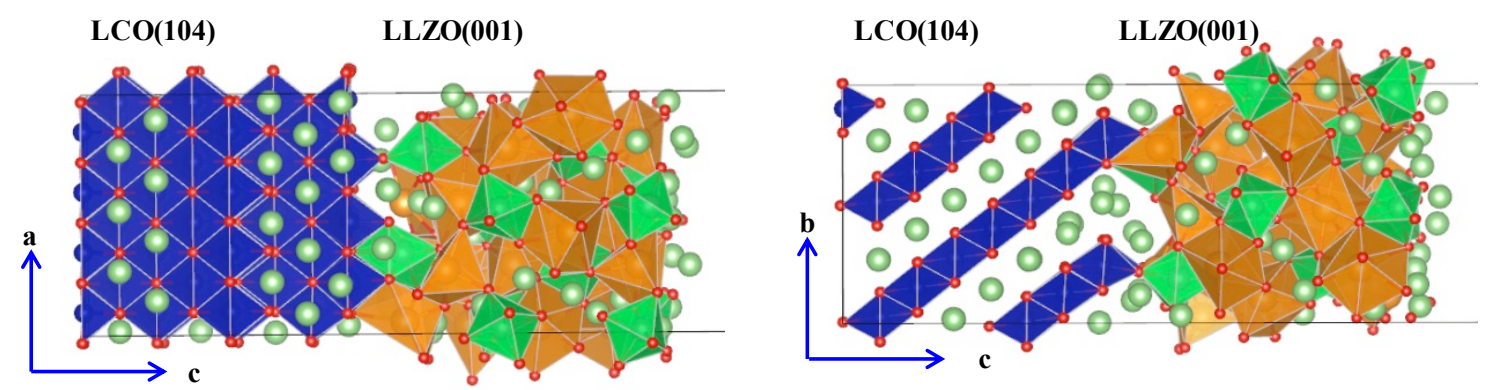
Figure S13 Top and side views of slab models of LCO(104)/LLZO(001) interface.
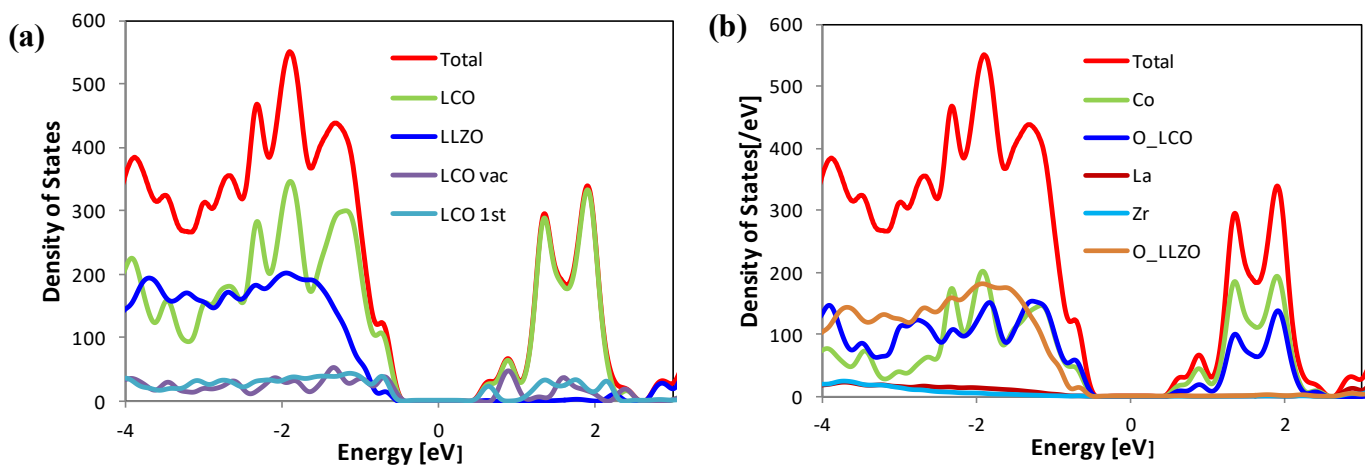

Figure S14. (a) Total and PDOS of LCO(104)/LLZO(001) interface model. (b) PDOS of atom species of LCO(104)/LLZO(001) interface model.

In Fig.S14, we show the total and partial DOS of LCO(104)/LLZO(001) interface model. Fig.S14.(a) show the PDOSs of LCO and LLZO slabs, where LCO_vac is the first LCO layer facing to the vacuum side, and LCO_1st is the first LCO layer facing to the LLZO slab. Fig.S14(b) show the PDOS of atom species of LCO(104)/LLZO(001) interface. O_LCO and O_LLZO indicate the PDOS of oxygen in LCO and that of LLZO, respectively.

In Fig.S15, we show the (a) the lowest (most stable) and (b) high energy $\left(0.024 \mathrm{eV} / \AA^{2}\right.$ higher than most stable structure) structure of interface structure of LCO(104)/LLZO(001) interface in our calculates interfaces. In Table S9, we show the Li vacancy formation energies corresponding to Fig.S15.

(a)

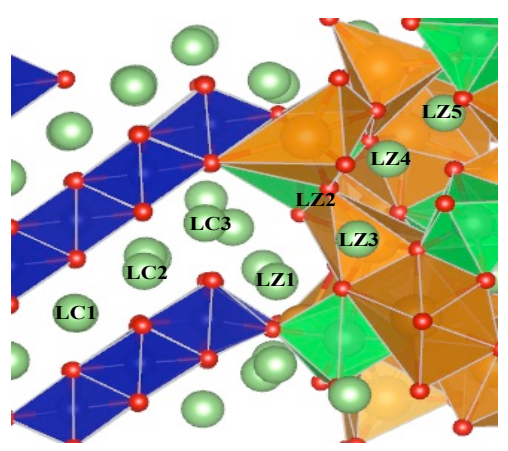

(b)

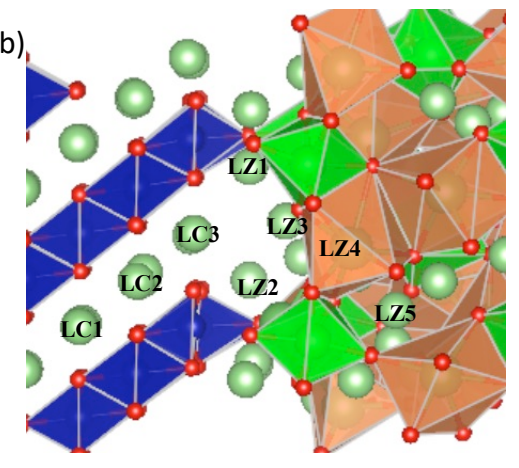

Figure S15. Structure of (a) the lowest and (b) high energy models of LCO(104)/LLZO(001) interface and the sites where we calculated Li vacancy formation energy.. 
Table S9. Li vacancy formation energies of each $\mathrm{Li}$ site where LC1 LC3 are Li site in LCO and LZ1 LZ4 are those in LLZO. The symbols of Li sites (LC1 LZ4 ) are corresponding to Fig. S15.

\begin{tabular}{|l|l|l|l|l|l|l|l|l|}
\hline & LC1 & LC2 & LC3 & LZ1 & LZ2 & LZ3 & LZ4 & LZ5 \\
\hline (a) & 3.36 & 3.17 & 3.43 & 2.51 & 2.60 & 3.04 & 2.92 & 2.89 \\
\hline (b) & 3.28 & 3.20 & 2.87 & 2.03 & 2.72 & 2.22 & 2.63 & 2.66 \\
\hline
\end{tabular}

In the interface model (b) with high energy, the Li sites with low vacancy formation energy such as LZ1 and LZ3 in Table S9 are increased. In LCO(104)/LLZO(001) interface, some of $\mathrm{LaO}_{8}$ complex in LLZO are broken at the interface by stoichiometric reason and it would disturb the atomic structure. Then, the LLZO interface tends to generate the rather high energy interface structure with electrode.

(a)

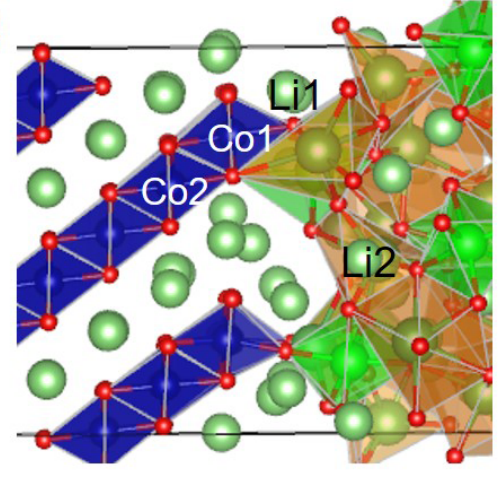

(b)

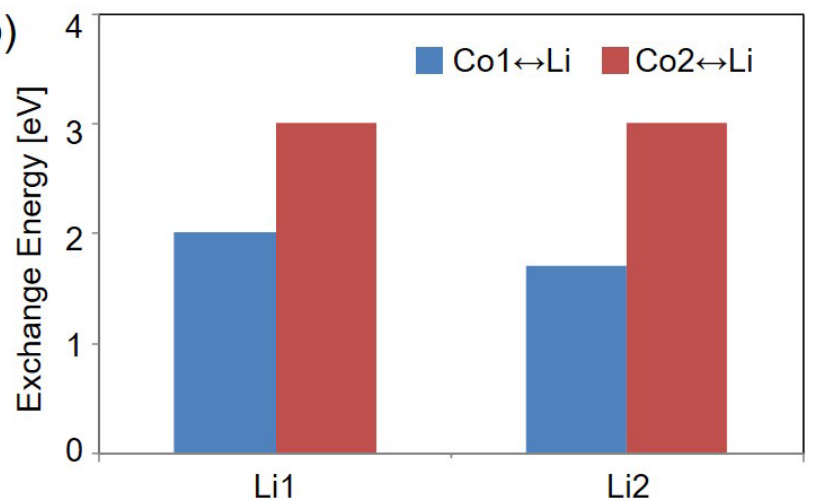

Figure S16. (a)Structure of the $\operatorname{LCO}(104) / \operatorname{LLZO}(001)$ interface (upper panel) and its corresponding (b) $\mathrm{Co} \leftrightarrow \mathrm{Li}$ mutual cation exchange reaction energies (lower panel). The positive values of exchange energies indicate endothermic reaction. 


\section{S6. LTO/LPS interface}

One of slab models of LTO(111)/LPS(010) interface we calculated are given in Fig. S17. The LTO(111)/LPS(010) slab model is composed of 1056 atoms including 672 and 384 atoms in LTO and LPS phase, respectively. The lattice constant of LPS is set to that of LCO. The misfit parameter of $\operatorname{LCO}(104) / \mathrm{LTO}(111)$ interface is $4.9 \%$. In Table S10, we show the misfit parameters and the repeated number of LTO and LPS units which construct the interface models. The LTO(111) and $\operatorname{LPS}(010)$ units are $11.907 \times 10.311 \times 14.58\left(168\right.$ atom) and $13.02 \times 8.161 \times 6.234$ (32 atom) $\AA^{3}$ orthorhombic cell, respectively.

Table S10. The repeated numbers of LTO and LPS units in the slab model and the misfit parameter ( $\mu$ ) of the calculated interface.

\begin{tabular}{|l|l|l|l|}
\hline Interface model & LTO(111) unit & LPS $(010)$ unit & $\mu(\%)$ \\
\hline LTO(111)/LPS(010) & $1 \times 4 \times 1$ & $3 \times 2 \times 2$ & 4.9 \\
\hline
\end{tabular}

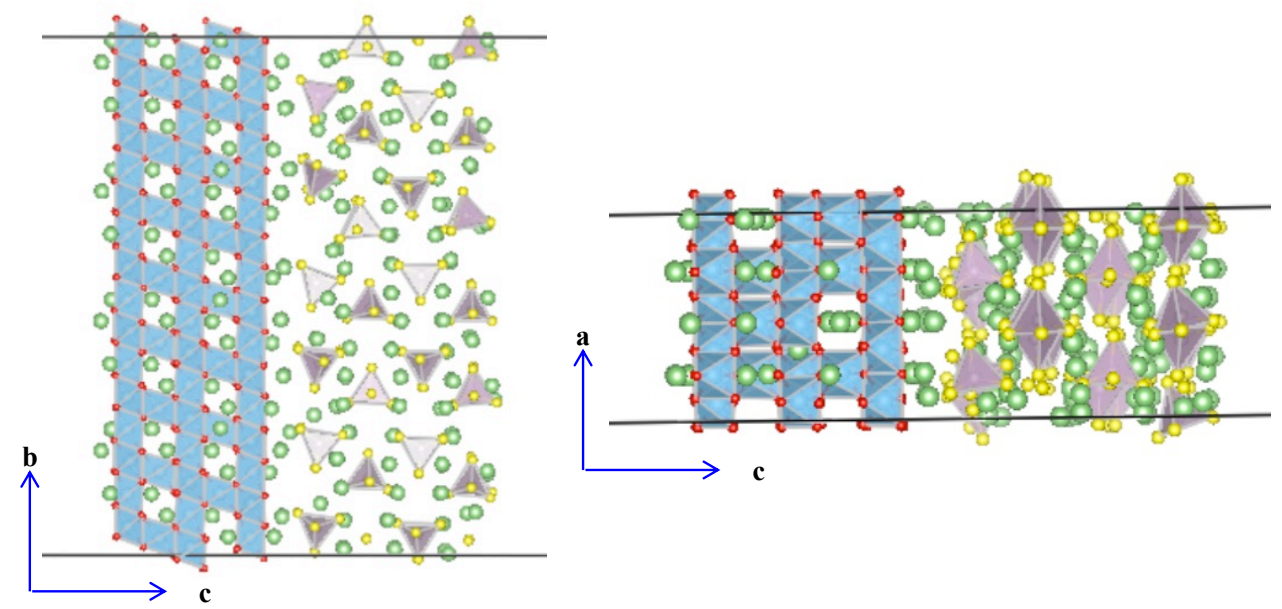

Figure S17. Top and side views of slab models of LTO(111)/LPS(010) interface.

Fig.S18 show the Li sites that we have calculated Li vacancy formation energies in one of slab models of LTO(111)/LPS(010) interface which has the most stable energy in our calculated 4 slab models of LTO(111)/LPS(010). The Li vacancy formation energies of each Li sites corresponding to FigS.18 are given in Table S11. 


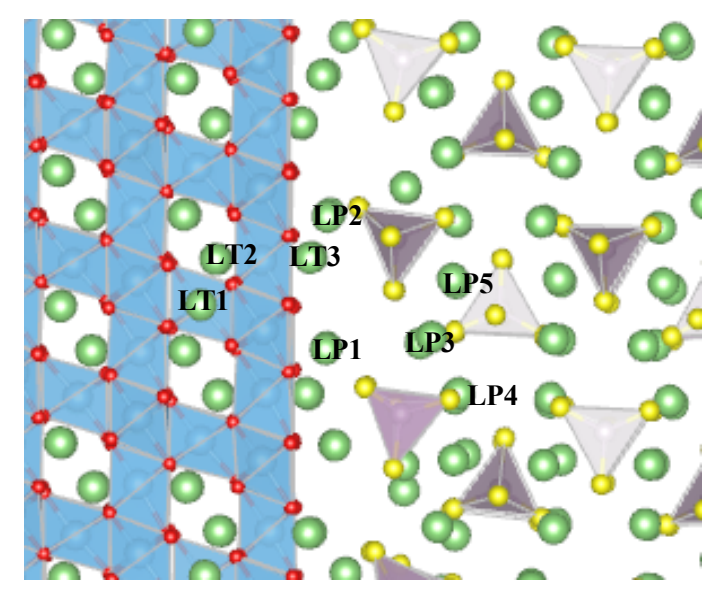

Figure S18. Typical structure of LTO(111)/LPS(010) interface and the sites where we calculated Li vacancy formation energy.

Table S11. Li vacancy formation energies of each Li site where LT1 LT3 are Li site in LTO and LP1 LP5 are those in LPS. The symbols of Li sites (LC1 LP5) are corresponding to Figure S17.

\begin{tabular}{|l|l|l|l|l|l|l|l|l|}
\hline & LT1 & LT2 & LT3 & LP1 & LP2 & LP3 & LP4 & LP5 \\
\hline$(\mathrm{eV})$ & 3.30 & 3.80 & 3.60 & 3.03 & 2.97 & 2.95 & 2.97 & 3.05 \\
\hline
\end{tabular}

Fig,S19, we show the structure of the LTO(111)/LPS(010) interface and their corresponding cation exchange energies in Fig.S20. The Ti $\leftrightarrow$ P cation exchange reaction at the LTO(111)/LPS(010) are endothermic and the reaction is less likely to occur.

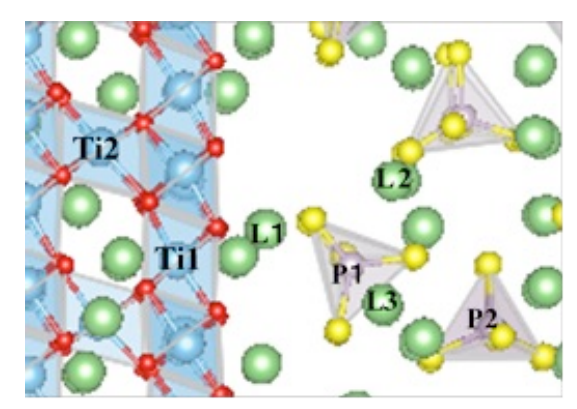

Figure S19. The sites where we calculated cation exchange energies in LTO(111)/LPS(010) interface. 

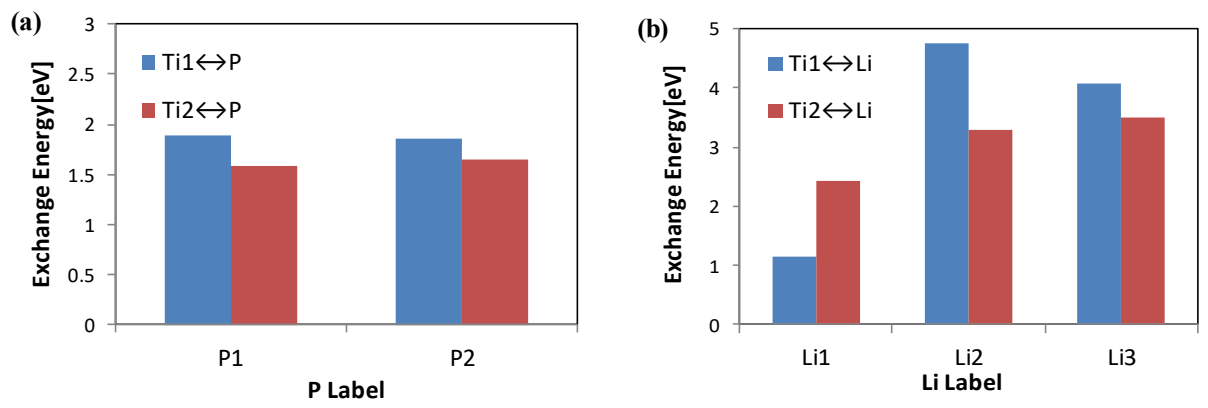

Figure S20. The reaction energies of (a) $\mathrm{Ti} \leftrightarrow \mathrm{P}$ and (b) $\mathrm{Ti} \leftrightarrow \mathrm{Li}$ cation exchange reaction energies, where the atom indexes corresponds to Fig.S19. The positive values of exchange energies correspond to endothermic reaction.

\section{S7. LCO/LTO interface}

One of slab models of $\mathrm{LCO}(104) / \mathrm{LTO}(111)$ interface we calculated are given in Fig. S21. The LCO(104)/LTO(111) slab model is composed of 424 atoms including 256 and 168 atoms in LCO and LTO phase, respectively. For LCO(104)/LTO(111) interface, we calculated 3 different interface models and took average of Li vacancy formation energy. The lattice constants of LTO are set to that of LCO. The misfit parameter of $\operatorname{LCO}(104) / \mathrm{LTO}(111)$ interface is $7.5 \%$. In Table S12, we show the misfit parameters and the repeated number of LTO and LPS units which construct the interface models. The $\mathrm{LCO}(104)$ and $\mathrm{LTO}(111)$ units are $2.815 \times 5.700 \times 8.012$ (18 atom), and $11.907 \times 10.311 \times 14.58$ (168 atom) $\AA^{3}$ orthorhombic cell, respectively.

Table S12. The repeated numbers of LCO and LTO units in the slab model and the misfit parameter $(\mu)$ of the calculated interface.

\begin{tabular}{|l|l|l|l|}
\hline Interface model & $\mathrm{LCO}(104)$ unit & LTO (111) unit & $\mu(\%)$ \\
\hline $\mathrm{LCO}(104) / \mathrm{LTO}(111)$ & $4 \times 2 \times 2$ & $1 \times 1 \times 1$ & 7.5 \\
\hline
\end{tabular}
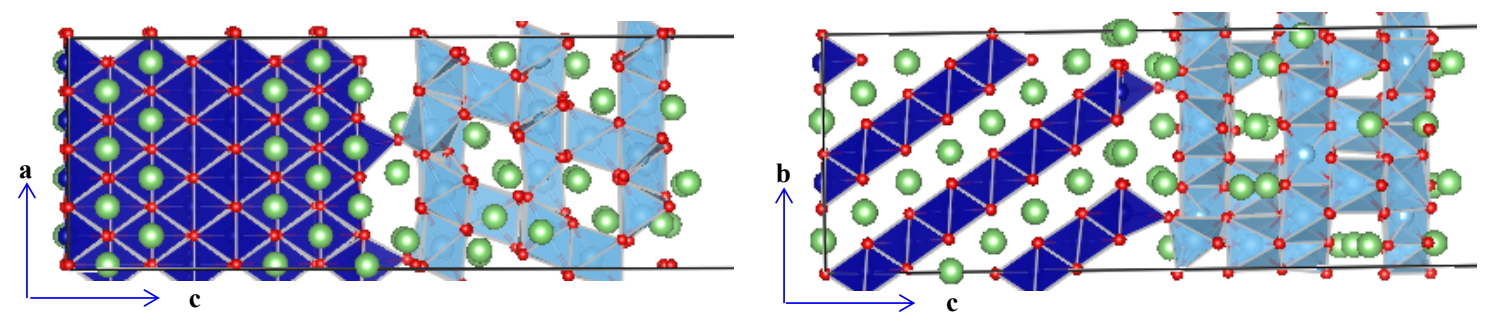

Figure S21. Typical structure of LTO(111)/LPS(010) interface and the sites where we calculated Li vacancy formation energy. 
In $\mathrm{LCO}(104) / \mathrm{LTO}(111)$ boundary, some of outermost $\mathrm{Co}$ atoms at $\mathrm{LCO}$ surface formed $\mathrm{CoO}_{6}$ octahedrons by sharing the $\mathrm{O}$ atoms in $\mathrm{TiO}_{6}$ tetrahedrons in $\mathrm{LTO}$ region.

We show the distribution of $\mathrm{Li}$ sites divided by their vacancy formation energies at the LCO(104)/LTO(111) interface in Fig.S22. The frequency of appearance of Li sites with low vacancy formation energy is very low at $\mathrm{LCO}(104) / \mathrm{LTO}(111)$ interface. The Li site with vacancy formation energy below $2.5 \mathrm{eV}$ did not appear. In Fig.S22, we show the most stable structure of $\mathrm{LCO}(104) / \mathrm{LTO}(111)$ interface in 3 different interface structures, and in Table S13, we show the Li vacancy formation energy corresponding to the Fig.S23.

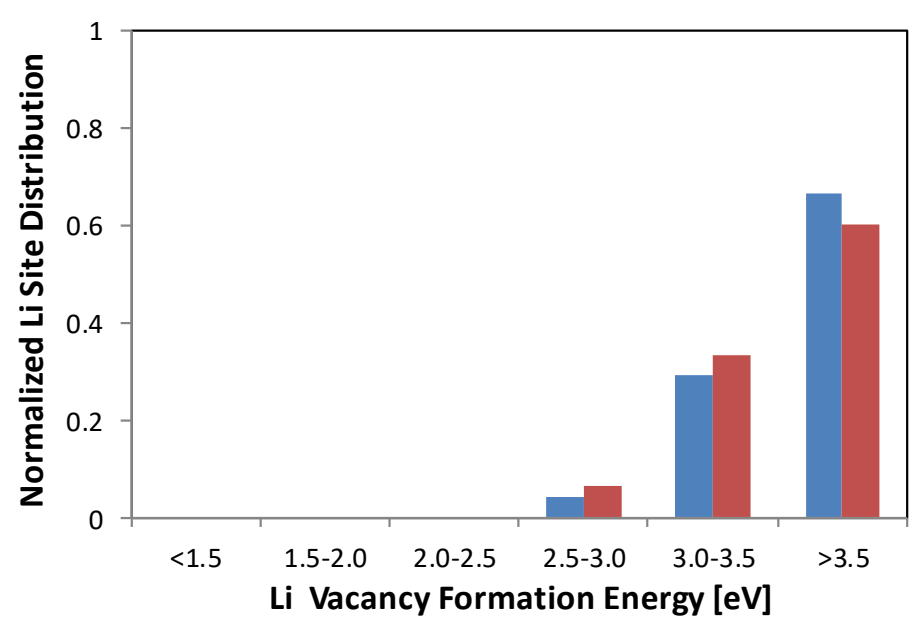

Figure S22. The distributions of $\mathrm{Li}$ sites divided by their $\mathrm{Li}$ vacancy formation energies at LCO(104)/LTO(111) interface. The vacancy formation energies of $24 \mathrm{Li}$ sites in 3 different interface structures are calculated. The distributions are normalized by the total number of calculated Li sites. The blue bar graphs denote distribution of all the calculated Li sites in LCO/LTO and red bar graphs are those of calculated Li sites located in LTO region.

Fig.S23 shows the Li sites that we have calculated Li vacancy formation energies in one of slab models of LCO(104)/LTO(111) interface which has the most stable energy in our calculated 3 slab models of $\mathrm{LCO}(104) / \mathrm{LTO}(111)$. The Li vacancy formation energies of each Li sites corresponding to FigS.23 are given in Table S13. 


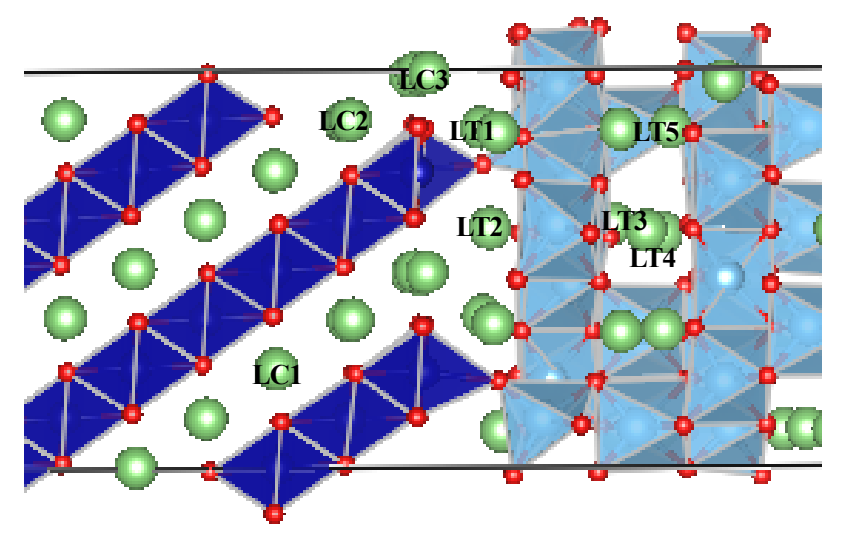

Figure S23. Typical structure of LCO(104)/LTO(111) interface and the sites where we calculated Li vacancy formation energy.

Table S13. Li vacancy formation energies of each Li site where LC1 LC3 are Li site in LCO and LT1 LT5 are those in LTO. The symbols of Li sites (LC1 LT5 ) are corresponding to Figure S22.

\begin{tabular}{|l|l|l|l|l|l|l|l|l|}
\hline & LC1 & LC2 & LC3 & LT1 & LT2 & LT3 & LT4 & LT5 \\
\hline$(\mathrm{eV})$ & 3.74 & 3.71 & 3.64 & 3.20 & 3.75 & 3.90 & 3.12 & 3.73 \\
\hline
\end{tabular}

\section{References}

[S1] Mercier, R.; Malugani, J.-P.; Fahys, B.; Robert, G.; Douglade, J. Structure du tetrathiophosphate de lithium. Acta Crystallogr. B 1982, 38, 1887-1890.

[S2] van Elp, J.; Wieland, J. L.; Eskes, H.; Kuipler, P.; Sawatzky, G. A.; de Groot, F. M. F.; Turner, T. S. Electronic structure of CoO, Li-doped CoO, and $\mathrm{LiCoO}_{2}$. Phys. Rev. B 1991, 44, 6090-6103.

[S3] Deschanvres, A.; Raveau, B.; Sekkal, Z. Mise en evidence et etude cristallographique d'une nouvelle solution solide de type spinelle $\mathrm{Li}_{1+\mathrm{x}} \mathrm{Ti}_{2-\mathrm{x}} \mathrm{O}_{4} 0 \leqslant \mathrm{x} \leqslant 0,33_{3}$. Mater. Res. Bull. 1971, 6, 699-704.

[S4] Yakubovich, O. V.; Urusov, V. S. Electron density distribution in lithiophosphatite $\mathrm{Li}_{3} \mathrm{PO}_{4}$; crystallochemical features of orthophosphates with hexagonal close packing. Kristallografiya 1997, 42, 301-308.

[S5] Geiger, C. A.; Alekseev, E.; Lazic, B.; Fisch, M.; Ambruster, T.; Langner, R.; Fechtelkord, M.; Kim, N.; Pettke, T.; Weppner, W. Crystal Chemistry and Stability of " $\mathrm{Li}_{7} \mathrm{La}_{3} \mathrm{Zr}_{2} \mathrm{O}_{12}$ " Garnet: A Fast Lithium-Ion Conductor. Inorg. Chem. 2011, 50, 1089-1097.

[S6] Liu, L. M.; Wang, S. Q.; Ye, H. Q. First-principles study of metal/nitride polar interfaces: 
Ti/TiN. Surf. Interface Anal. 2003, 35, 835-841.

[S7] Martin, L.; Vallverdu, G.; Martinez, H.; Le Cras, F.; Baraille, I. First principles calculations of solid-solid interfaces: an application to conversion materials for lithium-ion batteries. J. Mater. Chem. 2012, 22, 22063-22071.

[S8] Butler, K. T.; Gautam, G. S.; Cenepa, P.; Designing interfaces in energy materials applications with-first-principles calculations. npj Comput. Mater. 2019, 5, 19 